Acta Crystallographica Section E

Structure Reports

Online

ISSN 1600-5368

\section{2-(4-Isopropylbenzylidene)propanoic acid}

\section{Niaz Muhammad, ${ }^{a}$ M. Nawaz Tahir, ${ }^{b *}$ Saqib Ali, ${ }^{a}$ Zia-ur-Rehman $^{\mathrm{a}}$ and Muhammad Akram Kashmiric}

aDepartment of Chemistry, Quaid-i-Azam University, Islamabad 45320, Pakistan, ${ }^{\mathbf{b}}$ Department of Physics, University of Sargodha, Sagrodha, Pakistan, and 'Department of Chemistry, Government College University, Lahore, Pakistan Correspondence e-mail: dmntahir_uos@yahoo.com

Received 21 April 2008; accepted 4 July 2008

Key indicators: single-crystal X-ray study; $T=296 \mathrm{~K}$; mean $\sigma(\mathrm{C}-\mathrm{C})=0.004 \AA$; $R$ factor $=0.054 ; w R$ factor $=0.158 ;$ data-to-parameter ratio $=20.7$.

The two molecules in the asymmetric unit of the title compound, $\mathrm{C}_{13} \mathrm{H}_{16} \mathrm{O}_{2}$, form dimers through $\mathrm{O}-\mathrm{H} \cdots \mathrm{O}$ hydrogen bonding, resulting in $R_{2}^{2}(8)$ rings. Each carboxyl O atom is involved in interamolecular $\mathrm{C}-\mathrm{H} \cdots \mathrm{O}$ hydrogen bonds, forming five-membered rings. There exist dissimilar dihedral angles within the two molecules, for example the carboxylate and isopropyl groups make dihedral angles of $59.6(4)$ and $71.7(3)^{\circ}$ in the two molecules. There are no intermolecular $\pi$ interactions.

\section{Related literature}

For related literature, see: Burt (2004); Hertog et al. (1995); Ma \& Hayes (2004); Muhammad et al. (2007).

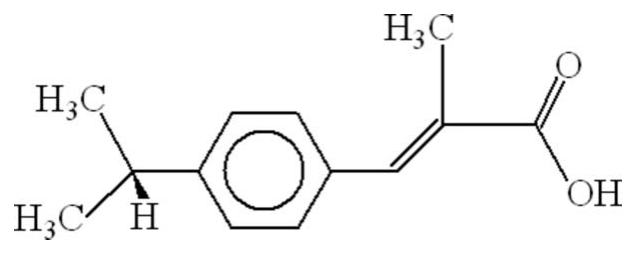

\section{Experimental}

Crystal data
$\mathrm{C}_{13} \mathrm{H}_{16} \mathrm{O}_{2}$
$M_{r}=204.26$
Triclinic, $P \overline{1}$
$a=9.8406(4) \AA$
$b=10.5739(4) \AA$
$c=11.9142(5) \AA$
$\alpha=96.330(2)^{\circ}$
$\beta=98.486(3)^{\circ}$

Data collection

Bruker Kappa APEXII CCD diffractometer

Absorption correction: multi-scan (SADABS; Bruker, 2005)

$T_{\min }=0.977, T_{\max }=0.986$

Refinement

$R\left[F^{2}>2 \sigma\left(F^{2}\right)\right]=0.053$

$w R\left(F^{2}\right)=0.157$

$S=1.04$

6000 reflections

290 parameters
23066 measured reflections 6000 independent reflections 2803 reflections with $I>3 \sigma(I)$ $R_{\text {int }}=0.033$

Table 1

Hydrogen-bond geometry $\left(\AA{ }^{\circ}\right)$.

\begin{tabular}{lllll}
\hline$D-\mathrm{H} \cdots A$ & $D-\mathrm{H}$ & $\mathrm{H} \cdots A$ & $D \cdots A$ & $D-\mathrm{H} \cdots A$ \\
\hline $\mathrm{O} 1-\mathrm{H} 1 \cdots \mathrm{O} 4^{\mathrm{i}}$ & $0.94(4)$ & $1.71(4)$ & $2.644(4)$ & $175(3)$ \\
$\mathrm{O} 3-\mathrm{H} 3 A \cdots \mathrm{O} 2^{\mathrm{ii}}$ & $0.93(4)$ & $1.71(4)$ & $2.631(3)$ & $169(3)$ \\
$\mathrm{C} 3-\mathrm{H} 3 \cdots \mathrm{O} 1$ & $0.96(3)$ & $2.35(2)$ & $2.707(4)$ & $101.2(16)$ \\
$\mathrm{C} 13-\mathrm{H} 13 A \cdots \mathrm{O} 2$ & 0.96 & 2.28 & $2.759(4)$ & 110 \\
$\mathrm{C} 16-\mathrm{H} 16 \cdots \mathrm{O} 3$ & $0.91(3)$ & $2.31(2)$ & $2.698(4)$ & $105.1(18)$ \\
$\mathrm{C} 26-\mathrm{H} 26 A \cdots \mathrm{O} 4$ & 0.96 & 2.30 & $2.770(4)$ & 110 \\
\hline
\end{tabular}

Symmetry codes: (i) $x, y, z+1$; (ii) $x, y, z-1$.

Data collection: $A P E X 2$ (Bruker, 2007); cell refinement: APEX2; data reduction: $S A I N T$ (Bruker, 2007); program(s) used to solve structure: SHELXS97 (Sheldrick, 2008); program(s) used to refine structure: SHELXL97 (Sheldrick, 2008); molecular graphics: ORTEP-3 for Windows (Farrugia, 1997) and PLATON (Spek, 2003); software used to prepare material for publication: Win $G X$ (Farrugia, 1999) and PLATON.

The authors acknowledge the Higher Education Commission, Islamabad, Pakistan, for funding the purchase of the diffractometer and for financial support to $\mathrm{NM}$ for $\mathrm{PhD}$ studies under the Indigenous Scholarship Scheme.

Supplementary data and figures for this paper are available from the IUCr electronic archives (Reference: JH2064).

\title{
References
}

Bruker (2005). SADABS. Bruker AXS Inc. Madison, Wisconsin, USA. Bruker (2007). APEX2 and SAINT. Bruker AXS Inc. Madison, Wisconsin, USA.

Burt, S. (2004). Int. J. Food Microbiol. 94, 223-253.

Farrugia, L. J. (1997). J. Appl. Cryst. 30, 565.

Farrugia, L. J. (1999). J. Appl. Cryst. 32, 837-838.

Hertog, M. G., Kromhout, D., Aravanis, C., Blackburn, H., Buzina, R., Fidanza, F., Giampaoli, S., Jansen, A., Menotti, A. \& Nedeljkovic, S. (1995). Arch. Intern. Med. 155, 381-386.

Ma, G. \& Hayes, S. E. (2004). J. Labelled Compd Radiopharm. 47, 895-901. Muhammad, N., Zia-ur-Rehman, A. S. \& Meetsma, A. (2007). Acta Cryst. E63, o2174-02175.

Sheldrick, G. M. (2008). Acta Cryst. A64, 112-122.

Spek, A. L. (2003). J. Appl. Cryst. 36, 7-13. 


\section{supporting information}

Acta Cryst. (2008). E64, o1456 [doi:10.1107/S1600536808020801]

\section{2-(4-Isopropylbenzylidene)propanoic acid}

\section{Niaz Muhammad, M. Nawaz Tahir, Saqib Ali, Zia-ur-Rehman and Muhammad Akram Kashmiri}

\section{S1. Comment}

Cinnamic acids and their derivatives are widely used chemicals in a variety of fields (Ma \& Hayes, 2004). They posses antibacterial, antifungal and parasite fighting abilities (Burt, 2004). A derivative of cinnamic acid is an important pharmaceutical for high blood pressure and stroke prevention and possess antitumour activity (Hertog et al., 1995).

The crystal structure of 3-(4-Bromophenyl)-2-methylacrylic acid (Muhammad et al., 2007) has been reported. The title compound (I) have a replacement of Br-atom with isopropyl at the same position. The ligand has been prepared to synthesize various organotin complexes.

The crystallographic asymmetric unit consists of two ligands. These two ligands form dimers through $\mathrm{O}-\mathrm{H} \cdots \mathrm{O}$ hydrogen bonding with each other by completing a $R_{2}{ }^{2}(8)$ rings (Table 1, Fig 2). Each O-atom of carboxylate ligands is involved in interamolecular $\mathrm{H}$-bonds of $\mathrm{C}-\mathrm{H} \cdots \mathrm{O}$ type forming five-membered rings. The bond distances in the benzene ring (C4-C9) have values in the range 1.372 (4)-1.390 (4) $\AA$, whereas in (C17-C22) its range is 1.374 (4)-1.384 (4) $\AA$. The bond angle $(\mathrm{C} 11-\mathrm{C} 10-\mathrm{C} 12)$ of isopropyl moiety is $111.2(3)^{\circ}$, whereas the same for $(\mathrm{C} 24-\mathrm{C} 23-\mathrm{C} 25)$ is $109.8(3)^{\circ}$. There exist a dissimilar dihedral angles within the two moieties. The dihedral angles of the isopropyl moieties $(\mathrm{C} 10 / \mathrm{C} 11 / \mathrm{C} 12)$ and $(\mathrm{C} 23 / \mathrm{C} 24 / \mathrm{C} 25)$ with their adjacent benzene rings $(\mathrm{C} 4-\mathrm{C} 9)$ and $(\mathrm{C} 17-\mathrm{C} 22)$ have values of $86.80(13)^{\circ}$ and $85.06(13)^{\circ}$, respectively. The dihedral angles of the moieties $(\mathrm{C} 2 / \mathrm{C} 3 / \mathrm{C} 13)$ and $(\mathrm{C} 15 / \mathrm{C} 16 / \mathrm{C} 26)$ with their adjacent carboxylate moities $(\mathrm{C} 1 / \mathrm{O} 1 / \mathrm{O} 2)$ and $(\mathrm{C} 14 / \mathrm{O} 3 / \mathrm{O} 4)$ have values of $7.65(51)^{\circ}$ and $7.06(43)^{\circ}$, respectively, whereas with benzene rings $(\mathrm{C} 4-\mathrm{C} 9)$ and $(\mathrm{C} 17-\mathrm{C} 22)$, the values of their dihedral angles is $32.18(23)^{\circ}$ and $34.49(20)^{\circ}$, respectively. The dihedral angle between the benzene rings of two ligands is $83.34(9)^{\circ}$. There does not exist any kind of $\pi$-interaction.

\section{S2. Experimental}

Compound (I) was prepared according to the reported procedure in literature (Muhammad et al., 2007). A mixture of 4isopropylbenzaldehyde (10 mmol, $1.51 \mathrm{ml})$, methylmalonic acid $(2.36 \mathrm{~g}, 20 \mathrm{mmol})$ and piperidine $(20 \mathrm{mmol}, 1.98 \mathrm{ml})$ in pyridine $(12.5 \mathrm{ml})$ solution was heated on a steam-bath for $24 \mathrm{~h}$. The reaction mixture was cooled and added to a mixture of $25 \mathrm{ml}$ of concentrated $\mathrm{HCl}$ and $50 \mathrm{~g}$ of ice. The precipitate formed in the acidified mixture was filtered off and washed with ice-cold water. The product was recrystallized from ethanol. The yield was $80 \%$.

\section{S3. Refinement}

$\mathrm{H}$ atoms were positioned geometrically, with $\mathrm{C}-\mathrm{H}=0.93$, and $0.96 \AA$ for aromatic and methyl $\mathrm{H}$, and constrained to ride on their parent atoms, while the coordinates of all other $\mathrm{H}$-atoms were refined. The $\mathrm{H}$-atoms were treated as isotropic with $\mathrm{U}_{\text {iso }}(\mathrm{H})=\mathrm{xU}_{\mathrm{eq}}(\mathrm{C}, \mathrm{O})$, where $\mathrm{x}=1.5$ for methyl $\mathrm{H}$, and $\mathrm{x}=1.2$ for all other $\mathrm{H}$ atoms. 

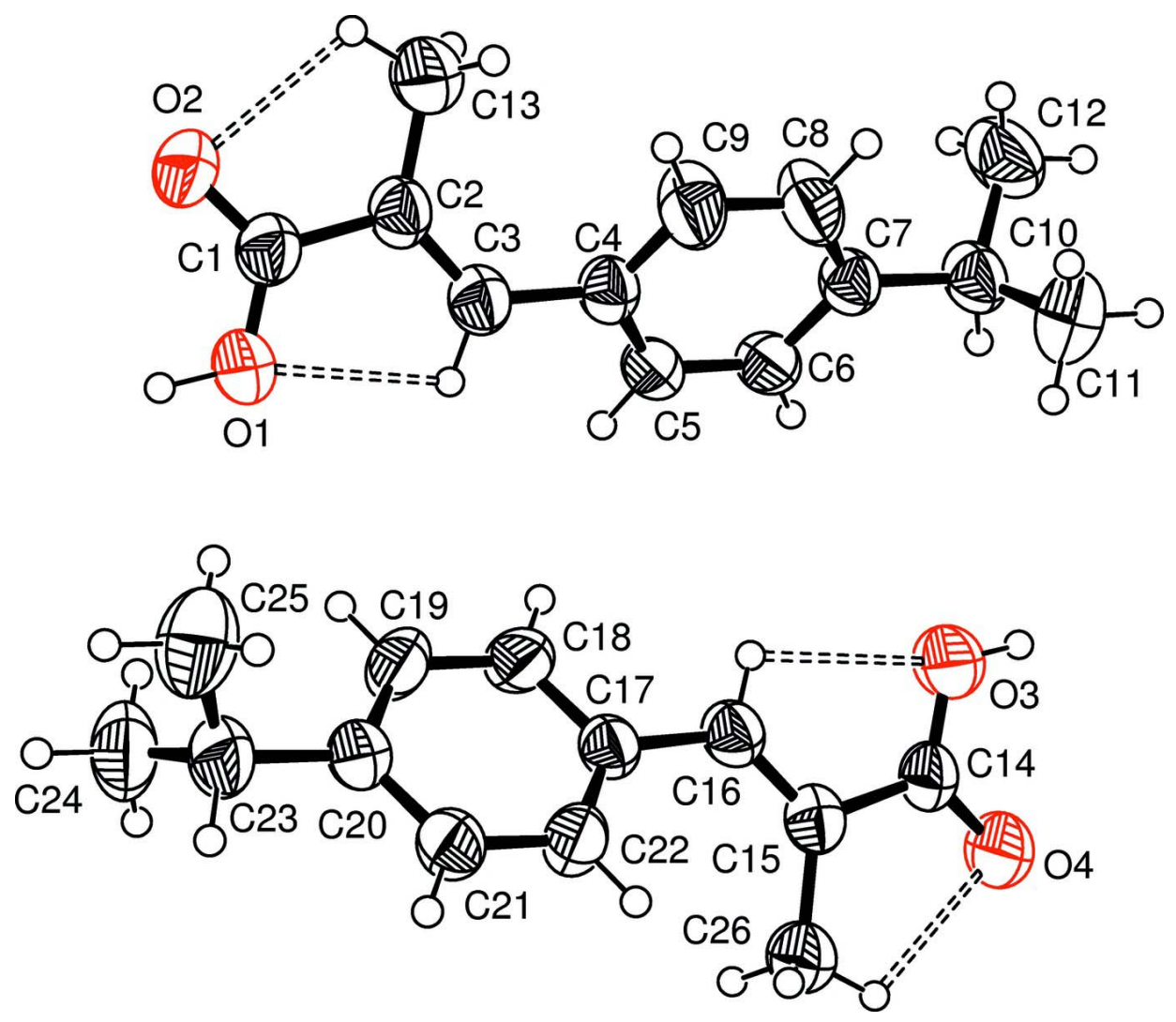

\section{Figure 1}

ORTEP drawing of the title compound, $\left(\mathrm{C}_{13} \mathrm{H}_{16} \mathrm{O}_{2}\right)_{2}$ with the atom numbering scheme. The thermal ellipsoids are drawn at the $50 \%$ probability level. H-atoms are shown by small circles of arbitrary radii. The intramolecular H-bonds are shown by doted lines. 


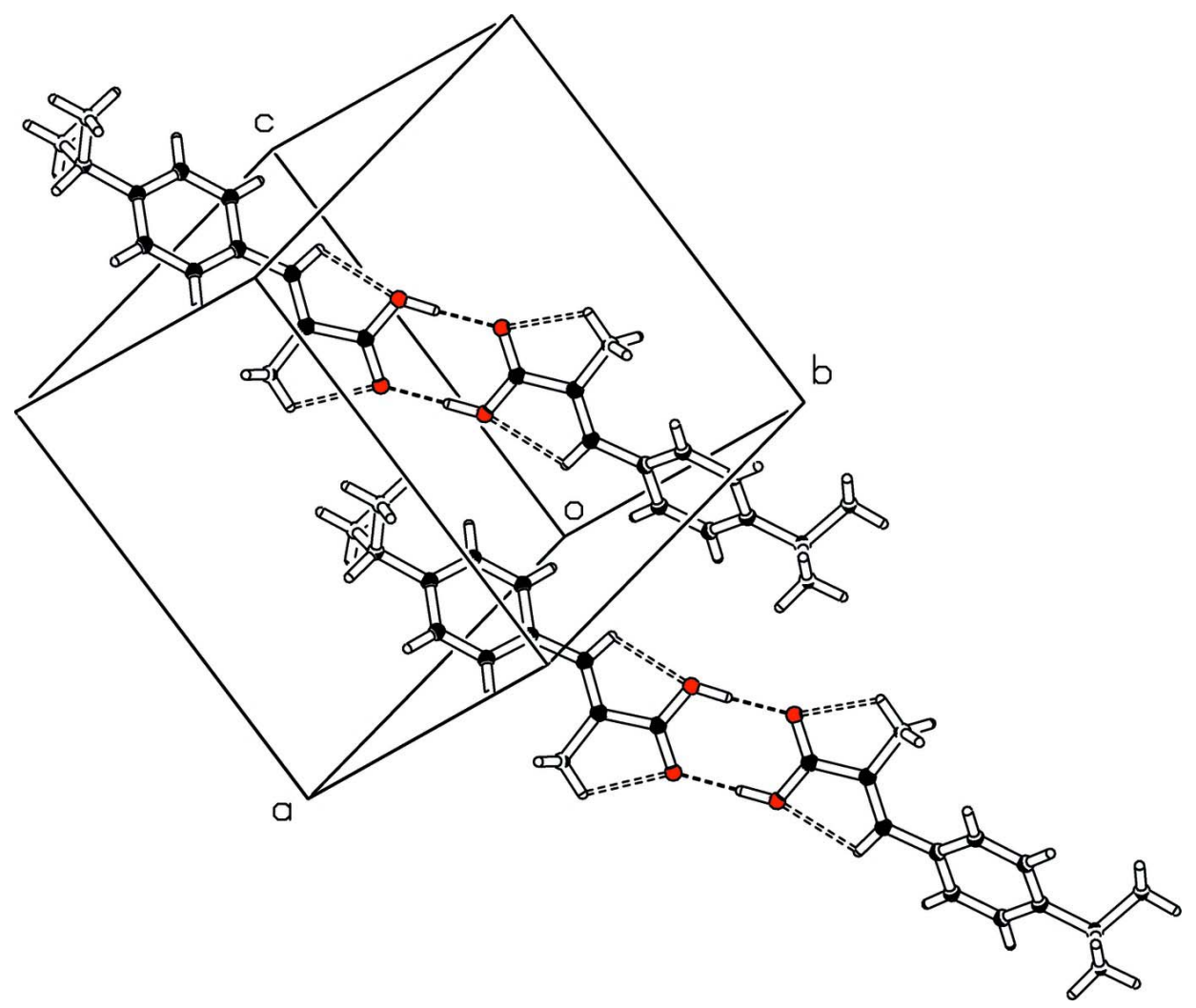

\section{Figure 2}

The partial packing figure (PLATON: Spek, 2003) which shows the dimeric nature of the compound.

\section{2-(4-Isopropylbenzylidene)propanoic acid}

\section{Crystal data}

$\mathrm{C}_{13} \mathrm{H}_{16} \mathrm{O}_{2}$

$M_{r}=204.26$

Triclinic, $P \overline{1}$

Hall symbol: -P 1

$a=9.8406(4) \AA$

$b=10.5739$ (4) $\AA$

$c=11.9142(5) \AA$

$\alpha=96.330(2)^{\circ}$

$\beta=98.486(3)^{\circ}$

$\gamma=104.497(2)^{\circ}$

$V=1172.99(8) \AA^{3}$

Data collection

\section{Bruker Kappa APEXII CCD} diffractometer

Radiation source: fine-focus sealed tube

Graphite monochromator

Detector resolution: 7.4 pixels $\mathrm{mm}^{-1}$

$\omega$ scans

Absorption correction: multi-scan

(SADABS; Bruker, 2005)

$T_{\min }=0.977, T_{\max }=0.986$
$Z=4$

$F(000)=440$

$D_{\mathrm{x}}=1.157 \mathrm{Mg} \mathrm{m}^{-3}$

Mo $K \alpha$ radiation radiation, $\lambda=0.71073 \AA$

Cell parameters from 2803 reflections

$\theta=2.2-28.8^{\circ}$

$\mu=0.08 \mathrm{~mm}^{-1}$

$T=296 \mathrm{~K}$

Prismatic, colourless

$0.30 \times 0.18 \times 0.12 \mathrm{~mm}$

23066 measured reflections

6000 independent reflections

2803 reflections with $I>3 \sigma(I)$

$R_{\text {int }}=0.033$

$\theta_{\text {max }}=28.8^{\circ}, \theta_{\text {min }}=2.2^{\circ}$

$h=-13 \rightarrow 13$

$k=-13 \rightarrow 14$

$l=-15 \rightarrow 16$ 


\section{Refinement}

Refinement on $F^{2}$

Least-squares matrix: full

$R\left[F^{2}>2 \sigma\left(F^{2}\right)\right]=0.053$

$w R\left(F^{2}\right)=0.157$

$S=1.04$

6000 reflections

290 parameters

0 restraints

Primary atom site location: structure-invariant direct methods

Secondary atom site location: difference Fourier map
Hydrogen site location: inferred from neighbouring sites

$\mathrm{H}$ atoms treated by a mixture of independent and constrained refinement

$w=1 /\left[\sigma^{2}\left(F_{\mathrm{o}}^{2}\right)+(0.0998 P)^{2}+0.3929 P\right]$ where $P=\left(F_{\mathrm{o}}{ }^{2}+2 F_{\mathrm{c}}{ }^{2}\right) / 3$

$(\Delta / \sigma)_{\max }=0.002$

$\Delta \rho_{\max }=0.28$ e $\AA^{-3}$

$\Delta \rho_{\text {min }}=-0.23$ e $\AA^{-3}$

Extinction correction: empirical, $\mathrm{Fc}^{*}=\mathrm{kFc}\left[1+0.001 \times \mathrm{xc}^{2} \lambda^{3} / \sin (2 \theta)\right]^{-1 / 4}$

Extinction coefficient: $0.020(3)$

\section{Special details}

Geometry. Bond distances, angles etc. have been calculated using the rounded fractional coordinates. All su's are estimated from the variances of the (full) variance-covariance matrix. The cell e.s.d.'s are taken into account in the estimation of distances, angles and torsion angles

Refinement. Refinement of $F^{2}$ against ALL reflections. The weighted $R$-factor $w R$ and goodness of fit $S$ are based on $F^{2}$, conventional $R$-factors $R$ are based on $F$, with $F$ set to zero for negative $F^{2}$. The threshold expression of $F^{2}>\sigma\left(F^{2}\right)$ is used only for calculating $R$-factors $(\mathrm{gt})$ etc. and is not relevant to the choice of reflections for refinement. $R$-factors based on $F^{2}$ are statistically about twice as large as those based on $F$, and $R$-factors based on ALL data will be even larger.

Fractional atomic coordinates and isotropic or equivalent isotropic displacement parameters $\left(\AA^{2}\right)$

\begin{tabular}{|c|c|c|c|c|}
\hline & $x$ & $y$ & $z$ & $U_{\text {iso }} * / U_{\text {eq }}$ \\
\hline $\mathrm{O} 1$ & $0.4946(3)$ & $0.6966(3)$ & 0.41038 (19) & $0.0929(11)$ \\
\hline $\mathrm{O} 2$ & $0.3929(3)$ & $0.8025(3)$ & $0.52698(18)$ & $0.0859(10)$ \\
\hline $\mathrm{C} 1$ & $0.4167(3)$ & $0.7736(3)$ & $0.4302(3)$ & $0.0604(10)$ \\
\hline $\mathrm{C} 2$ & $0.3538(3)$ & $0.8277(3)$ & $0.3319(2)$ & $0.0558(10)$ \\
\hline $\mathrm{C} 3$ & 0.3665 & $0.7807(3)$ & $0.2271(2)$ & $0.0572(10)$ \\
\hline $\mathrm{C} 4$ & $0.3150(3)$ & $0.8149(3)$ & $0.1158(2)$ & $0.0529(9)$ \\
\hline $\mathrm{C} 5$ & $0.2805(3)$ & 0.7198 & $0.0194(2)$ & $0.0567(10)$ \\
\hline C6 & $0.2312(3)$ & $0.7457(3)$ & $-0.0873(2)$ & $0.0575(9)$ \\
\hline $\mathrm{C} 7$ & $0.2160(3)$ & $0.8681(3)$ & $-0.1039(2)$ & $0.0536(9)$ \\
\hline $\mathrm{C} 8$ & $0.2534(4)$ & $0.9643(3)$ & $-0.0083(3)$ & $0.0816(13)$ \\
\hline C9 & $0.3021(4)$ & $0.9397(3)$ & $0.0992(3)$ & $0.0802(13)$ \\
\hline $\mathrm{C} 10$ & $0.1631(3)$ & $0.8967(3)$ & -0.2215 & $0.0652(11)$ \\
\hline $\mathrm{C} 11$ & $0.2802(4)$ & $0.9901(4)$ & -0.2652 & $0.0981(16)$ \\
\hline $\mathrm{C} 12$ & $0.0319(4)$ & $0.9478(4)$ & $-0.2243(3)$ & $0.0919(17)$ \\
\hline $\mathrm{C} 13$ & $0.2769(4)$ & 0.9280 & $0.3617(3)$ & $0.0818(12)$ \\
\hline $\mathrm{O} 3$ & $0.5215(3)$ & $0.7354(3)$ & $-0.28826(18)$ & $0.0829(9)$ \\
\hline $\mathrm{O} 4$ & $0.6264(3)$ & $0.6312(3)$ & $-0.40400(18)$ & $0.0887(10)$ \\
\hline $\mathrm{C} 14$ & $0.6113(3)$ & $0.6697(3)$ & $-0.3062(2)$ & $0.0559(10)$ \\
\hline $\mathrm{C} 15$ & $0.6987(3)$ & $0.6413(3)$ & $-0.2046(2)$ & $0.0531(9)$ \\
\hline $\mathrm{C} 16$ & $0.6664(3)$ & $0.6713(3)$ & $-0.1024(2)$ & $0.0538(9)$ \\
\hline $\mathrm{C} 17$ & 0.7335 & $0.6564(2)$ & $0.0123(2)$ & $0.0490(9)$ \\
\hline $\mathrm{C} 18$ & $0.6479(3)$ & 0.6330 & $0.0942(2)$ & $0.0640(10)$ \\
\hline C19 & 0.7024 & $0.6191(3)$ & $0.2041(2)$ & $0.0697(13)$ \\
\hline $\mathrm{C} 20$ & $0.8461(3)$ & $0.6321(3)$ & $0.2382(2)$ & $0.0548(9)$ \\
\hline
\end{tabular}




$\begin{array}{lllll}\text { C21 } & 0.9313(3) & 0.6562(3) & 0.1571(2) & 0.0561(9) \\ \text { C22 } & 0.8773(3) & 0.6688(3) & 0.0464(2) & 0.0562(9) \\ \text { C23 } & 0.9076(4) & 0.6231(3) & 0.3602(3) & 0.0708(1) \\ \text { C24 } & 0.8264(4) & 0.5008(4) & 0.4022(3) & 0.0926(16) \\ \text { C25 } & 0.9114(4) & 0.7468(4) & 0.4414(3) & 0.0945(16) \\ \text { C26 } & 0.8123(3) & 0.5764(3) & -0.2296(3) & 0.0682(11) \\ \text { H1 } & 0.537(4) & 0.669(4) & 0.476(3) & 0.1113^{*} \\ \text { H3 } & 0.410(3) & 0.709(3) & 0.218(2) & 0.0686^{*} \\ \text { H5 } & 0.29082 & 0.63593 & 0.02693 & 0.0680^{*} \\ \text { H6 } & 0.20748 & 0.67847 & -0.14988 & 0.0690^{*} \\ \text { H8 } & 0.24547 & 1.04865 & -0.01676 & 0.0975^{*} \\ \text { H9 } & 0.32671 & 1.00736 & 0.16152 & 0.0961^{*} \\ \text { H10 } & 0.140(3) & 0.809(3) & -0.279(3) & 0.0784^{*} \\ \text { H11A } & 0.24320 & 1.00662 & -0.33965 & 0.1470^{*} \\ \text { H11B } & 0.35749 & 0.95085 & -0.27047 & 0.1470^{*} \\ \text { H11C } & 0.31399 & 1.07189 & -0.21301 & 0.1470^{*} \\ \text { H12A } & 0.00214 & 0.96492 & -0.30028 & 0.1381^{*} \\ \text { H12B } & 0.05422 & 1.02807 & -0.17077 & 0.1381^{*} \\ \text { H12C } & -0.04376 & 0.88278 & -0.20377 & 0.1381^{*} \\ \text { H13A } & 0.28465 & 0.94509 & 0.44353 & 0.1227^{*} \\ \text { H13B } & 0.17804 & 0.89513 & 0.32631 & 0.1227^{*} \\ \text { H13C } & 0.31834 & 1.00840 & 0.33437 & 0.1227^{*} \\ \text { H3A } & 0.470(4) & 0.748(3) & -0.356(3) & 0.0995^{*} \\ \text { H16 } & 0.587(3) & 0.701(3) & -0.102(2) & 0.0645^{*} \\ \text { H18 } & 0.55108 & 0.62637 & 0.07461 & 0.0768^{*} \\ \text { H19 } & 0.64129 & 0.60065 & 0.25632 & 0.0836^{*} \\ \text { H21 } & 1.02841 & 0.66423 & 0.17740 & 0.0674^{*} \\ \text { H22 } & 0.93853 & 0.68585 & -0.00598 & 0.0674^{*} \\ \text { H23 } & 1.009(4) & 0.624(3) & 0.362(3) & 0.0851^{*} \\ \text { H24A } & 0.86976 & 0.49923 & 0.47953 & 0.1391^{*} \\ \text { H24B } & 0.82917 & 0.42321 & 0.35347 & 0.1391^{*} \\ \text { H24C } & 0.72907 & 0.50259 & 0.40018 & 0.1391^{*} \\ \text { H25A } & 0.95096 & 0.73954 & 0.51830 & 0.1420^{*} \\ \text { H25B } & 0.81621 & 0.75538 & 0.43888 & 0.1420^{*} \\ \text { H25C } & 0.96945 & 0.82322 & 0.41810 & 0.1420^{*} \\ \text { H26A } & 0.81376 & 0.56746 & -0.31044 & 0.1023^{*} \\ \text { H26B } & 0.79248 & 0.49055 & -0.20633 & 0.1023^{*} \\ \text { H26C } & 0.90349 & 0.62961 & -0.18800 & 0.1023^{*}\end{array}$

Atomic displacement parameters $\left(\AA^{2}\right)$

\begin{tabular}{lllllll}
\hline & $U^{11}$ & $U^{22}$ & $U^{33}$ & $U^{12}$ & $U^{13}$ & $U^{23}$ \\
\hline O1 & $0.124(2)$ & $0.133(2)$ & $0.0530(14)$ & $0.0852(18)$ & $0.0161(13)$ & $0.0295(13)$ \\
O2 & $0.1026(17)$ & $0.129(2)$ & $0.0474(13)$ & $0.0638(15)$ & $0.0173(12)$ & $0.0229(12)$ \\
C1 & $0.0587(17)$ & $0.0766(19)$ & $0.0484(18)$ & $0.0235(14)$ & $0.0068(13)$ & $0.0122(14)$ \\
C2 & $0.0520(16)$ & $0.0657(17)$ & $0.0501(17)$ & $0.0173(13)$ & $0.0042(12)$ & $0.0140(13)$ \\
C3 & $0.0579(17)$ & $0.0641(17)$ & $0.0521(18)$ & $0.0204(14)$ & $0.0070(13)$ & $0.0150(13)$ \\
C4 & $0.0521(15)$ & $0.0582(16)$ & $0.0479(16)$ & $0.0154(12)$ & $0.0041(12)$ & $0.0113(12)$
\end{tabular}


supporting information

\begin{tabular}{lllllll} 
C5 & $0.0657(17)$ & $0.0561(16)$ & $0.0539(18)$ & $0.0277(13)$ & $0.0077(13)$ & $0.0108(13)$ \\
C6 & $0.0669(17)$ & $0.0578(16)$ & $0.0485(16)$ & $0.0256(13)$ & $0.0022(13)$ & $0.0015(12)$ \\
C7 & $0.0585(16)$ & $0.0545(15)$ & $0.0463(16)$ & $0.0175(12)$ & $0.0013(12)$ & $0.0073(12)$ \\
C8 & $0.132(3)$ & $0.0526(17)$ & $0.059(2)$ & $0.0344(18)$ & $-0.0045(19)$ & $0.0109(14)$ \\
C9 & $0.127(3)$ & $0.0553(17)$ & $0.0504(19)$ & $0.0250(17)$ & $-0.0045(18)$ & $0.0022(13)$ \\
C10 & $0.082(2)$ & $0.0626(18)$ & $0.0511(18)$ & $0.0279(16)$ & $-0.0016(15)$ & $0.0081(14)$ \\
C11 & $0.119(3)$ & $0.114(3)$ & $0.070(2)$ & $0.033(2)$ & $0.028(2)$ & $0.033(2)$ \\
C12 & $0.096(3)$ & $0.101(3)$ & $0.086(3)$ & $0.050(2)$ & $-0.006(2)$ & $0.021(2)$ \\
C13 & $0.096(2)$ & $0.100(2)$ & $0.060(2)$ & $0.051(2)$ & $0.0051(17)$ & $0.0127(17)$ \\
O3 & $0.0924(16)$ & $0.1221(19)$ & $0.0495(13)$ & $0.0610(15)$ & $0.0026(11)$ & $0.0178(12)$ \\
O4 & $0.121(2)$ & $0.1225(19)$ & $0.0434(13)$ & $0.0694(16)$ & $0.0134(12)$ & $0.0186(12)$ \\
C14 & $0.0642(17)$ & $0.0628(16)$ & $0.0431(17)$ & $0.0216(14)$ & $0.0066(13)$ & $0.0128(12)$ \\
C15 & $0.0584(16)$ & $0.0521(14)$ & $0.0479(16)$ & $0.0146(12)$ & $0.0059(12)$ & $0.0105(12)$ \\
C16 & $0.0567(16)$ & $0.0578(16)$ & $0.0483(17)$ & $0.0213(13)$ & $0.0034(13)$ & $0.0093(12)$ \\
C17 & $0.0559(16)$ & $0.0495(14)$ & $0.0431(15)$ & $0.0198(11)$ & $0.0046(12)$ & $0.0069(11)$ \\
C18 & $0.0521(16)$ & $0.098(2)$ & $0.0483(17)$ & $0.0340(15)$ & $0.0073(13)$ & $0.0095(15)$ \\
C19 & $0.0619(19)$ & $0.113(3)$ & $0.0445(17)$ & $0.0346(17)$ & $0.0169(14)$ & $0.0206(16)$ \\
C20 & $0.0565(17)$ & $0.0635(16)$ & $0.0466(16)$ & $0.0220(13)$ & $0.0048(13)$ & $0.0109(12)$ \\
C21 & $0.0468(15)$ & $0.0680(17)$ & $0.0530(17)$ & $0.0167(12)$ & $0.0035(13)$ & $0.0116(13)$ \\
C22 & $0.0537(16)$ & $0.0681(17)$ & $0.0469(16)$ & $0.0138(13)$ & $0.0112(12)$ & $0.0131(13)$ \\
C23 & $0.0678(19)$ & $0.100(2)$ & $0.0496(18)$ & $0.0323(18)$ & $0.0030(15)$ & $0.0207(16)$ \\
C24 & $0.132(3)$ & $0.093(3)$ & $0.057(2)$ & $0.038(2)$ & $0.008(2)$ & $0.0242(18)$ \\
C25 & $0.110(3)$ & $0.099(3)$ & $0.057(2)$ & $0.010(2)$ & $-0.0022(19)$ & $0.0044(18)$ \\
C26 & $0.080(2)$ & $0.077(2)$ & $0.0547(19)$ & $0.0349(16)$ & $0.0105(15)$ & $0.0105(14)$ \\
& & & & & & \\
\hline
\end{tabular}

Geometric parameters $\left(\AA,{ }^{\circ}\right)$

\begin{tabular}{llll}
\hline $\mathrm{O} 1-\mathrm{C} 1$ & $1.277(4)$ & $\mathrm{C} 13-\mathrm{H} 13 \mathrm{~A}$ & 0.9600 \\
$\mathrm{O} 2-\mathrm{C} 1$ & $1.231(4)$ & $\mathrm{C} 13-\mathrm{H} 13 \mathrm{~B}$ & 0.9600 \\
$\mathrm{O} 1-\mathrm{H} 1$ & $0.94(4)$ & $\mathrm{C} 13-\mathrm{H} 13 \mathrm{C}$ & 0.9600 \\
$\mathrm{O} 3-\mathrm{C} 14$ & $1.281(4)$ & $\mathrm{C} 14-\mathrm{C} 15$ & $1.482(4)$ \\
$\mathrm{O} 4-\mathrm{C} 14$ & $1.234(3)$ & $\mathrm{C} 15-\mathrm{C} 16$ & $1.328(4)$ \\
$\mathrm{O} 3-\mathrm{H} 3 \mathrm{~A}$ & $0.93(4)$ & $\mathrm{C} 15-\mathrm{C} 26$ & $1.499(4)$ \\
$\mathrm{C} 1-\mathrm{C} 2$ & $1.481(4)$ & $\mathrm{C} 16-\mathrm{C} 17$ & $1.470(3)$ \\
$\mathrm{C} 2-\mathrm{C} 13$ & $1.491(5)$ & $\mathrm{C} 17-\mathrm{C} 18$ & $1.384(4)$ \\
$\mathrm{C} 2-\mathrm{C} 3$ & $1.327(3)$ & $\mathrm{C} 17-\mathrm{C} 22$ & $1.382(4)$ \\
$\mathrm{C} 3-\mathrm{C} 4$ & $1.465(4)$ & $\mathrm{C} 18-\mathrm{C} 19$ & $1.378(3)$ \\
$\mathrm{C} 4-\mathrm{C} 5$ & $1.380(4)$ & $\mathrm{C} 19-\mathrm{C} 20$ & $1.380(4)$ \\
$\mathrm{C} 4-\mathrm{C} 9$ & $1.390(4)$ & $\mathrm{C} 20-\mathrm{C} 21$ & $1.374(4)$ \\
$\mathrm{C} 5-\mathrm{C} 6$ & $1.374(4)$ & $\mathrm{C} 20-\mathrm{C} 23$ & $1.513(4)$ \\
$\mathrm{C} 6-\mathrm{C} 7$ & $1.372(4)$ & $\mathrm{C} 21-\mathrm{C} 22$ & $1.381(3)$ \\
$\mathrm{C} 7-\mathrm{C} 10$ & $1.511(4)$ & $\mathrm{C} 23-\mathrm{C} 24$ & $1.518(5)$ \\
$\mathrm{C} 7-\mathrm{C} 8$ & $1.379(4)$ & $\mathrm{C} 23-\mathrm{C} 25$ & $1.528(5)$ \\
$\mathrm{C} 8-\mathrm{C} 9$ & $1.376(5)$ & $\mathrm{C} 16-\mathrm{H} 16$ & $0.91(3)$ \\
$\mathrm{C} 10-\mathrm{C} 11$ & $1.515(5)$ & $\mathrm{C} 18-\mathrm{H} 18$ & 0.9300 \\
$\mathrm{C} 10-\mathrm{C} 12$ & $1.517(5)$ & $\mathrm{C} 19-\mathrm{H} 19$ & 0.9300 \\
$\mathrm{C} 3-\mathrm{H} 3$ & $0.96(3)$ & $\mathrm{C} 21-\mathrm{H} 21$ & 0.9300 \\
$\mathrm{C} 5-\mathrm{H} 5$ & 0.9300 & $\mathrm{C} 22-\mathrm{H} 22$ & 0.9300
\end{tabular}




\begin{tabular}{|c|c|c|c|}
\hline $\mathrm{C} 6-\mathrm{H} 6$ & 0.9300 & $\mathrm{C} 23-\mathrm{H} 23$ & $0.99(4)$ \\
\hline $\mathrm{C} 8-\mathrm{H} 8$ & 0.9300 & $\mathrm{C} 24-\mathrm{H} 24 \mathrm{~A}$ & 0.9600 \\
\hline C9- $\mathrm{H} 9$ & 0.9300 & $\mathrm{C} 24-\mathrm{H} 24 \mathrm{~B}$ & 0.9600 \\
\hline $\mathrm{C} 10-\mathrm{H} 10$ & $1.04(3)$ & $\mathrm{C} 24-\mathrm{H} 24 \mathrm{C}$ & 0.9600 \\
\hline $\mathrm{C} 11-\mathrm{H} 11 \mathrm{~A}$ & 0.9600 & $\mathrm{C} 25-\mathrm{H} 25 \mathrm{~A}$ & 0.9600 \\
\hline $\mathrm{C} 11-\mathrm{H} 11 \mathrm{~B}$ & 0.9600 & $\mathrm{C} 25-\mathrm{H} 25 \mathrm{~B}$ & 0.9600 \\
\hline $\mathrm{C} 11-\mathrm{H} 11 \mathrm{C}$ & 0.9600 & $\mathrm{C} 25-\mathrm{H} 25 \mathrm{C}$ & 0.9600 \\
\hline $\mathrm{C} 12-\mathrm{H} 12 \mathrm{C}$ & 0.9600 & $\mathrm{C} 26-\mathrm{H} 26 \mathrm{~A}$ & 0.9600 \\
\hline $\mathrm{C} 12-\mathrm{H} 12 \mathrm{~B}$ & 0.9600 & $\mathrm{C} 26-\mathrm{H} 26 \mathrm{~B}$ & 0.9600 \\
\hline $\mathrm{C} 12-\mathrm{H} 12 \mathrm{~A}$ & 0.9600 & $\mathrm{C} 26-\mathrm{H} 26 \mathrm{C}$ & 0.9600 \\
\hline $\mathrm{O} 1 \cdots \mathrm{O} 4^{\mathrm{i}}$ & $2.644(4)$ & $\mathrm{H} 5 \cdots \mathrm{C} 17^{\mathrm{iv}}$ & 3.0200 \\
\hline $\mathrm{O} 2 \cdots \mathrm{O} 3^{\mathrm{i}}$ & $2.631(3)$ & H6 $\cdots$ H10 & 2.3100 \\
\hline $\mathrm{O} 2 \cdots \mathrm{C} 14^{\mathrm{i}}$ & $3.377(4)$ & $\mathrm{H} 6 \cdots \mathrm{H} 24 \mathrm{~B}^{\mathrm{iv}}$ & 2.4800 \\
\hline $\mathrm{O} 3 \cdots \mathrm{O} 2^{\mathrm{ii}}$ & $2.631(3)$ & $\mathrm{H} 8 \cdots \mathrm{H} 11 \mathrm{C}$ & 2.5500 \\
\hline $\mathrm{O} 4 \cdots \mathrm{C} 1^{\mathrm{ii}}$ & $3.382(4)$ & $\mathrm{H} 8 \cdots \mathrm{H} 12 \mathrm{~B}$ & 2.3800 \\
\hline $\mathrm{O} 4 \cdots \mathrm{O} 1^{\mathrm{ii}}$ & $2.644(4)$ & $\mathrm{H} 8 \cdots \mathrm{C} 16^{\mathrm{iii}}$ & 2.9900 \\
\hline $\mathrm{O} 1 \cdots \mathrm{H} 19$ & 2.7500 & $\mathrm{H} 8 \cdots \mathrm{C} 17^{\mathrm{iii}}$ & 3.0700 \\
\hline $\mathrm{O} 1 \cdots \mathrm{H} 3 \mathrm{~A}^{\mathrm{i}}$ & $2.83(4)$ & $\mathrm{H} 8 \cdots \mathrm{C} 12$ & 2.9000 \\
\hline $\mathrm{O} 1 \cdots \mathrm{H} 3$ & $2.35(2)$ & $\mathrm{H} 8 \cdots \mathrm{C} 11$ & 3.0400 \\
\hline $\mathrm{O} 2 \cdots \mathrm{H} 11 \mathrm{~B}^{\mathrm{i}}$ & 2.8400 & $\mathrm{H} 9 \cdots \mathrm{C} 13$ & 2.6900 \\
\hline $\mathrm{O} 2 \cdots \mathrm{H} 13 \mathrm{~A}$ & 2.2800 & $\mathrm{H} 9 \cdots \mathrm{C} 2$ & 2.9600 \\
\hline $\mathrm{O} 2 \cdots \mathrm{H} 3 \mathrm{~A}^{\mathrm{i}}$ & $1.71(4)$ & $\mathrm{H} 9 \cdots \mathrm{O} 3^{\mathrm{iii}}$ & 2.8800 \\
\hline $\mathrm{O} 3 \cdots \mathrm{H} 9^{\mathrm{iii}}$ & 2.8800 & $\mathrm{H} 9 \cdots \mathrm{H} 13 \mathrm{C}$ & 2.0700 \\
\hline $\mathrm{O} 3 \cdots \mathrm{H} 16$ & $2.31(2)$ & 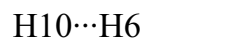 & 2.3100 \\
\hline $\mathrm{O} 3 \cdots \mathrm{H} 1^{\mathrm{ii}}$ & $2.86(4)$ & H11A $\cdots H 12 A$ & 2.4300 \\
\hline $\mathrm{O} 4 \cdots \mathrm{H} 1^{\mathrm{ii}}$ & $1.71(4)$ & $\mathrm{H} 11 \mathrm{~B} \cdots \mathrm{O} 2^{\mathrm{ii}}$ & 2.8400 \\
\hline $\mathrm{O} 4 \cdots \mathrm{H} 26 \mathrm{~A}$ & 2.3000 & $\mathrm{H} 11 \mathrm{C} \cdots \mathrm{C} 8$ & 2.8800 \\
\hline $\mathrm{C} 1 \cdots \mathrm{O} 4^{\mathrm{i}}$ & $3.382(4)$ & $\mathrm{H} 11 \mathrm{C} \cdots \mathrm{H} 8$ & 2.5500 \\
\hline C9 $\cdots$ C13 & $3.187(5)$ & $\mathrm{H} 12 \mathrm{~A} \cdots \mathrm{H} 13 \mathrm{~B}^{\mathrm{v}}$ & 2.5900 \\
\hline $\mathrm{C} 13 \cdots \mathrm{C} 9$ & $3.187(5)$ & H12A $\cdots H 11 \mathrm{~A}$ & 2.4300 \\
\hline $\mathrm{C} 14 \cdots \mathrm{O} 2^{\mathrm{ii}}$ & 3.377 (4) & $\mathrm{H} 12 \mathrm{~B} \cdots \mathrm{C} 8$ & 2.8100 \\
\hline $\mathrm{C} 22 \cdots \mathrm{C} 26$ & $3.246(4)$ & $\mathrm{H} 12 \mathrm{~B} \cdots \mathrm{H} 8$ & 2.3800 \\
\hline $\mathrm{C} 26 \cdots \mathrm{C} 22$ & $3.246(4)$ & $\mathrm{H} 13 \mathrm{~A} \cdots \mathrm{O} 2$ & 2.2800 \\
\hline $\mathrm{C} 1 \cdots \mathrm{H} 3 \mathrm{~A}^{\mathrm{i}}$ & $2.58(3)$ & $\mathrm{H} 13 \mathrm{~B} \cdots \mathrm{H} 25 \mathrm{C}^{\mathrm{vi}}$ & 2.4600 \\
\hline $\mathrm{C} 2 \cdots \mathrm{H} 9$ & 2.9600 & $\mathrm{H} 13 \mathrm{~B} \cdots \mathrm{H} 12 \mathrm{~A}^{\mathrm{v}}$ & 2.5900 \\
\hline $\mathrm{C} 3 \cdots \mathrm{H} 26 \mathrm{~B}^{\mathrm{iv}}$ & 2.8700 & $\mathrm{H} 13 \mathrm{C} \cdots \mathrm{C} 9$ & 2.7900 \\
\hline $\mathrm{C} 5 \cdots \mathrm{H} 18$ & 3.0700 & $\mathrm{H} 13 \mathrm{C} \cdots \mathrm{H} 9$ & 2.0700 \\
\hline $\mathrm{C} 8 \cdots \mathrm{H} 11 \mathrm{C}$ & 2.8800 & $\mathrm{H} 16 \cdots \mathrm{O} 3$ & $2.31(2)$ \\
\hline $\mathrm{C} 8 \cdots \mathrm{H} 12 \mathrm{~B}$ & 2.8100 & $\mathrm{H} 16 \cdots \mathrm{H} 18$ & 2.3700 \\
\hline C9 $\cdots \mathrm{H} 13 \mathrm{C}$ & 2.7900 & $\mathrm{H} 18 \cdots \mathrm{C} 5$ & 3.0700 \\
\hline $\mathrm{C} 11 \cdots \mathrm{H} 8$ & 3.0400 & $\mathrm{H} 18 \cdots \mathrm{H} 3$ & 2.5700 \\
\hline $\mathrm{C} 12 \cdots \mathrm{H} 8$ & 2.9000 & $\mathrm{H} 18 \cdots \mathrm{H} 5$ & 2.5700 \\
\hline $\mathrm{C} 13 \cdots \mathrm{H} 9$ & 2.6900 & $\mathrm{H} 18 \cdots \mathrm{H} 16$ & 2.3700 \\
\hline $\mathrm{C} 14 \cdots \mathrm{H} 1^{\mathrm{ii}}$ & $2.59(4)$ & $\mathrm{H} 19 \cdots \mathrm{O} 1$ & 2.7500 \\
\hline $\mathrm{C} 15 \cdots \mathrm{H} 22$ & 2.9900 & H19 ‥C24 & 2.8100 \\
\hline $\mathrm{C} 16 \cdots \mathrm{H} 8^{\mathrm{iii}}$ & 2.9900 & $\mathrm{H} 19 \cdots \mathrm{H} 24 \mathrm{C}$ & 2.2700 \\
\hline $\mathrm{C} 17 \cdots \mathrm{H} 5^{\text {iv }}$ & 3.0200 & $\mathrm{H} 21 \cdots \mathrm{H} 23$ & 2.3100 \\
\hline
\end{tabular}


$\mathrm{C} 17 \cdots \mathrm{H} 8^{\mathrm{iii}}$

$\mathrm{C} 19 \cdots \mathrm{H} 24 \mathrm{C}$

$\mathrm{C} 19 \cdots \mathrm{H} 25 \mathrm{~B}$

$\mathrm{C} 22 \cdots \mathrm{H} 26 \mathrm{C}$

$\mathrm{C} 24 \cdots \mathrm{H} 19$

$\mathrm{C} 26 \cdots \mathrm{H} 22$

$\mathrm{H} 1 \cdots \mathrm{O} 4^{\mathrm{i}}$

$\mathrm{H} 1 \cdots{ }^{\circ} 3^{i}$

$\mathrm{H} 1 \cdots \mathrm{C} 14^{\mathrm{i}}$

$\mathrm{H} 1 \cdots \mathrm{H} 3 \mathrm{~A}^{\mathrm{i}}$

$\mathrm{H} 3 \cdots \mathrm{H} 5$

$\mathrm{H} 3 \cdots \mathrm{O} 1$

$\mathrm{H} 3 \cdots \mathrm{H} 18$

$\mathrm{H} 3 \cdots \mathrm{H}^{2} 6 \mathrm{~B}^{\mathrm{iv}}$

$\mathrm{H} 3 \mathrm{~A} \cdots \mathrm{C} 1^{\text {ii }}$

$\mathrm{H} 3 \mathrm{~A}^{\cdots} \mathrm{O}^{\mathrm{ii}}$

$\mathrm{H} 3 \mathrm{~A} \cdots \mathrm{O} 2^{\mathrm{ii}}$

$\mathrm{H} 3 \mathrm{~A} \cdots \mathrm{H} 1^{1 i}$

$\mathrm{H} 5 \cdots \mathrm{H} 3$

$\mathrm{H} 5 \cdots \mathrm{H} 18$

$\mathrm{C} 1-\mathrm{O} 1-\mathrm{H} 1$

$\mathrm{C} 14-\mathrm{O} 3-\mathrm{H} 3 \mathrm{~A}$

$\mathrm{O} 1-\mathrm{C} 1-\mathrm{O} 2$

$\mathrm{O} 1-\mathrm{C} 1-\mathrm{C} 2$

$\mathrm{O} 2-\mathrm{C} 1-\mathrm{C} 2$

$\mathrm{C} 1-\mathrm{C} 2-\mathrm{C} 13$

$\mathrm{C} 1-\mathrm{C} 2-\mathrm{C} 3$

$\mathrm{C} 3-\mathrm{C} 2-\mathrm{C} 13$

$\mathrm{C} 2-\mathrm{C} 3-\mathrm{C} 4$

$\mathrm{C} 3-\mathrm{C} 4-\mathrm{C} 9$

$\mathrm{C} 3-\mathrm{C} 4-\mathrm{C} 5$

$\mathrm{C} 5-\mathrm{C} 4-\mathrm{C} 9$

$\mathrm{C} 4-\mathrm{C} 5-\mathrm{C} 6$

$\mathrm{C} 5-\mathrm{C} 6-\mathrm{C} 7$

$\mathrm{C} 8-\mathrm{C} 7-\mathrm{C} 10$

$\mathrm{C} 6-\mathrm{C} 7-\mathrm{C} 8$

$\mathrm{C} 6-\mathrm{C} 7-\mathrm{C} 10$

C7-C $8-\mathrm{C} 9$

$\mathrm{C} 4-\mathrm{C} 9-\mathrm{C} 8$

$\mathrm{C} 7-\mathrm{C} 10-\mathrm{C} 12$

$\mathrm{C} 7-\mathrm{C} 10-\mathrm{C} 11$

$\mathrm{C} 11-\mathrm{C} 10-\mathrm{C} 12$

$\mathrm{C} 2-\mathrm{C} 3-\mathrm{H} 3$

$\mathrm{C} 4-\mathrm{C} 3-\mathrm{H} 3$

$\mathrm{C} 6-\mathrm{C} 5-\mathrm{H} 5$

$\mathrm{C} 4-\mathrm{C} 5-\mathrm{H} 5$

C5-C6- 66
3.0700

2.7700

2.9200

2.8400

2.8100

2.7600

$1.71(4)$

2.86 (4)

2.59 (4)

$2.33(5)$

2.3500

2.35 (2)

2.5700

2.4900

$2.58(3)$

2.83 (4)

1.71 (4)

$2.33(5)$

2.3500

2.5700

115 (2)

113 (2)

$122.1(3)$

117.7 (3)

$120.2(3)$

115.4 (2)

$118.2(3)$

126.4 (3)

129.9 (3)

$124.2(3)$

$119.0(3)$

116.7 (3)

$121.8(3)$

$121.7(3)$

$121.6(3)$

116.7 (2)

$121.6(2)$

$122.2(3)$

120.8 (3)

$112.2(3)$

$111.5(3)$

$111.2(3)$

$118.7(14)$

$111.3(14)$

119.00

119.00

119.00
$\mathrm{H} 22 \cdots \mathrm{C} 15$

$\mathrm{H} 22 \cdots \mathrm{C} 26$

$\mathrm{H} 22 \cdots \mathrm{H} 26 \mathrm{C}$

$\mathrm{H} 23 \cdots \mathrm{H} 21$

$\mathrm{H} 24 \mathrm{~A} \cdots \mathrm{H} 25 \mathrm{~A}$

$\mathrm{H} 24 \mathrm{~A} \cdots \mathrm{H} 24 \mathrm{~A}^{\mathrm{vii}}$

$\mathrm{H} 24 \mathrm{~B} \cdots \mathrm{H} 6^{\text {iv }}$

$\mathrm{H} 24 \mathrm{C} \cdots \mathrm{C} 19$

$\mathrm{H} 24 \mathrm{C} \cdots \mathrm{H} 19$

$\mathrm{H} 24 \mathrm{C} \cdots \mathrm{H} 25 \mathrm{~B}$

$\mathrm{H} 25 \mathrm{~A} \cdots \mathrm{H} 24 \mathrm{~A}$

$\mathrm{H} 25 \mathrm{~B} \cdots \mathrm{C} 19$

$\mathrm{H} 25 \mathrm{~B} \cdots \mathrm{H} 24 \mathrm{C}$

$\mathrm{H} 25 \mathrm{C} \cdots \mathrm{H} 13 \mathrm{~B}^{\text {viii }}$

$\mathrm{H} 26 \mathrm{~A} \cdots \mathrm{O} 4$

$\mathrm{H} 26 \mathrm{~B} \cdots \mathrm{C}^{\text {iv }}$

$\mathrm{H} 26 \mathrm{~B} \cdots \mathrm{H}^{\text {iv }}$

$\mathrm{H} 26 \mathrm{C} \cdots \mathrm{C} 22$

$\mathrm{H} 26 \mathrm{C} \cdots \mathrm{H} 22$

$\mathrm{H} 13 \mathrm{~A}-\mathrm{C} 13-\mathrm{H} 13 \mathrm{C}$

$\mathrm{O} 3-\mathrm{C} 14-\mathrm{O} 4$

$\mathrm{O} 3-\mathrm{C} 14-\mathrm{C} 15$

$\mathrm{O} 4-\mathrm{C} 14-\mathrm{C} 15$

C14-C15-C16

C14-C15-C26

$\mathrm{C} 16-\mathrm{C} 15-\mathrm{C} 26$

$\mathrm{C} 15-\mathrm{C} 16-\mathrm{C} 17$

$\mathrm{C} 16-\mathrm{C} 17-\mathrm{C} 22$

$\mathrm{C} 18-\mathrm{C} 17-\mathrm{C} 22$

C16-C17-C18

C17-C18-C19

$\mathrm{C} 18-\mathrm{C} 19-\mathrm{C} 20$

$\mathrm{C} 19-\mathrm{C} 20-\mathrm{C} 21$

$\mathrm{C} 21-\mathrm{C} 20-\mathrm{C} 23$

$\mathrm{C} 19-\mathrm{C} 20-\mathrm{C} 23$

$\mathrm{C} 20-\mathrm{C} 21-\mathrm{C} 22$

$\mathrm{C} 17-\mathrm{C} 22-\mathrm{C} 21$

$\mathrm{C} 20-\mathrm{C} 23-\mathrm{C} 25$

$\mathrm{C} 24-\mathrm{C} 23-\mathrm{C} 25$

$\mathrm{C} 20-\mathrm{C} 23-\mathrm{C} 24$

$\mathrm{C} 15-\mathrm{C} 16-\mathrm{H} 16$

C17-C16-H16

C17-C18-H18

C19-C18-H18

C18-C19-H19

C20-C19-H19
2.9900

2.7600

2.1400

2.3100

2.4300

2.5300

2.4800

2.7700

2.2700

2.5600

2.4300

2.9200

2.5600

2.4600

2.3000

2.8700

2.4900

2.8400

2.1400

109.00

$122.0(3)$

117.7 (2)

$120.4(3)$

$117.6(3)$

$115.6(2)$

$126.8(3)$

130.1 (3)

$125.0(2)$

117.0 (2)

$117.9(3)$

121.7 (3)

$121.2(3)$

117.1 (2)

121.5 (3)

121.4 (3)

122.1 (3)

120.9 (3)

$110.6(3)$

109.8 (3)

$112.6(3)$

$116.4(15)$

$113.4(15)$

119.00

119.00

119.00

119.00 


\begin{tabular}{|c|c|c|c|}
\hline $\mathrm{C} 7-\mathrm{C} 6-\mathrm{H} 6$ & 119.00 & $\mathrm{C} 20-\mathrm{C} 21-\mathrm{H} 21$ & 119.00 \\
\hline $\mathrm{C} 9-\mathrm{C} 8-\mathrm{H} 8$ & 119.00 & $\mathrm{C} 22-\mathrm{C} 21-\mathrm{H} 21$ & 119.00 \\
\hline $\mathrm{C} 7-\mathrm{C} 8-\mathrm{H} 8$ & 119.00 & $\mathrm{C} 17-\mathrm{C} 22-\mathrm{H} 22$ & 120.00 \\
\hline $\mathrm{C} 4-\mathrm{C} 9-\mathrm{H} 9$ & 120.00 & $\mathrm{C} 21-\mathrm{C} 22-\mathrm{H} 22$ & 120.00 \\
\hline $\mathrm{C} 8-\mathrm{C} 9-\mathrm{H} 9$ & 120.00 & $\mathrm{C} 20-\mathrm{C} 23-\mathrm{H} 23$ & $107(2)$ \\
\hline $\mathrm{C} 11-\mathrm{C} 10-\mathrm{H} 10$ & $103.8(18)$ & $\mathrm{C} 24-\mathrm{C} 23-\mathrm{H} 23$ & $111.1(19)$ \\
\hline $\mathrm{C} 12-\mathrm{C} 10-\mathrm{H} 10$ & $110.3(17)$ & $\mathrm{C} 25-\mathrm{C} 23-\mathrm{H} 23$ & $105.4(19)$ \\
\hline $\mathrm{C} 7-\mathrm{C} 10-\mathrm{H} 10$ & $107.5(19)$ & $\mathrm{C} 23-\mathrm{C} 24-\mathrm{H} 24 \mathrm{~A}$ & 109.00 \\
\hline $\mathrm{C} 10-\mathrm{C} 11-\mathrm{H} 11 \mathrm{~B}$ & 109.00 & $\mathrm{C} 23-\mathrm{C} 24-\mathrm{H} 24 \mathrm{~B}$ & 109.00 \\
\hline $\mathrm{C} 10-\mathrm{C} 11-\mathrm{H} 11 \mathrm{C}$ & 109.00 & $\mathrm{C} 23-\mathrm{C} 24-\mathrm{H} 24 \mathrm{C}$ & 109.00 \\
\hline $\mathrm{H} 11 \mathrm{~A}-\mathrm{C} 11-\mathrm{H} 11 \mathrm{~B}$ & 110.00 & $\mathrm{H} 24 \mathrm{~A}-\mathrm{C} 24-\mathrm{H} 24 \mathrm{~B}$ & 109.00 \\
\hline $\mathrm{H} 11 \mathrm{~A}-\mathrm{C} 11-\mathrm{H} 11 \mathrm{C}$ & 110.00 & $\mathrm{H} 24 \mathrm{~A}-\mathrm{C} 24-\mathrm{H} 24 \mathrm{C}$ & 109.00 \\
\hline $\mathrm{H} 11 \mathrm{~B}-\mathrm{C} 11-\mathrm{H} 11 \mathrm{C}$ & 109.00 & $\mathrm{H} 24 \mathrm{~B}-\mathrm{C} 24-\mathrm{H} 24 \mathrm{C}$ & 109.00 \\
\hline $\mathrm{C} 10-\mathrm{C} 11-\mathrm{H} 11 \mathrm{~A}$ & 109.00 & $\mathrm{C} 23-\mathrm{C} 25-\mathrm{H} 25 \mathrm{~A}$ & 109.00 \\
\hline $\mathrm{C} 10-\mathrm{C} 12-\mathrm{H} 12 \mathrm{~A}$ & 109.00 & $\mathrm{C} 23-\mathrm{C} 25-\mathrm{H} 25 \mathrm{~B}$ & 109.00 \\
\hline $\mathrm{C} 10-\mathrm{C} 12-\mathrm{H} 12 \mathrm{C}$ & 109.00 & $\mathrm{C} 23-\mathrm{C} 25-\mathrm{H} 25 \mathrm{C}$ & 109.00 \\
\hline $\mathrm{H} 12 \mathrm{~A}-\mathrm{C} 12-\mathrm{H} 12 \mathrm{~B}$ & 109.00 & $\mathrm{H} 25 \mathrm{~A}-\mathrm{C} 25-\mathrm{H} 25 \mathrm{~B}$ & 109.00 \\
\hline $\mathrm{H} 12 \mathrm{~A}-\mathrm{C} 12-\mathrm{H} 12 \mathrm{C}$ & 109.00 & $\mathrm{H} 25 \mathrm{~A}-\mathrm{C} 25-\mathrm{H} 25 \mathrm{C}$ & 109.00 \\
\hline $\mathrm{H} 12 \mathrm{~B}-\mathrm{C} 12-\mathrm{H} 12 \mathrm{C}$ & 109.00 & $\mathrm{H} 25 \mathrm{~B}-\mathrm{C} 25-\mathrm{H} 25 \mathrm{C}$ & 109.00 \\
\hline $\mathrm{C} 10-\mathrm{C} 12-\mathrm{H} 12 \mathrm{~B}$ & 110.00 & $\mathrm{C} 15-\mathrm{C} 26-\mathrm{H} 26 \mathrm{~A}$ & 109.00 \\
\hline $\mathrm{C} 2-\mathrm{C} 13-\mathrm{H} 13 \mathrm{~A}$ & 110.00 & $\mathrm{C} 15-\mathrm{C} 26-\mathrm{H} 26 \mathrm{~B}$ & 109.00 \\
\hline $\mathrm{C} 2-\mathrm{C} 13-\mathrm{H} 13 \mathrm{C}$ & 109.00 & $\mathrm{C} 15-\mathrm{C} 26-\mathrm{H} 26 \mathrm{C}$ & 109.00 \\
\hline $\mathrm{H} 13 \mathrm{~A}-\mathrm{C} 13-\mathrm{H} 13 \mathrm{~B}$ & 109.00 & $\mathrm{H} 26 \mathrm{~A}-\mathrm{C} 26-\mathrm{H} 26 \mathrm{~B}$ & 109.00 \\
\hline $\mathrm{C} 2-\mathrm{C} 13-\mathrm{H} 13 \mathrm{~B}$ & 109.00 & $\mathrm{H} 26 \mathrm{~A}-\mathrm{C} 26-\mathrm{H} 26 \mathrm{C}$ & 109.00 \\
\hline $\mathrm{H} 13 \mathrm{~B}-\mathrm{C} 13-\mathrm{H} 13 \mathrm{C}$ & 109.00 & $\mathrm{H} 26 \mathrm{~B}-\mathrm{C} 26-\mathrm{H} 26 \mathrm{C}$ & 109.00 \\
\hline $\mathrm{O} 1-\mathrm{C} 1-\mathrm{C} 2-\mathrm{C} 3$ & $8.4(5)$ & $\mathrm{O} 3-\mathrm{C} 14-\mathrm{C} 15-\mathrm{C} 16$ & $8.1(4)$ \\
\hline $\mathrm{O} 1-\mathrm{C} 1-\mathrm{C} 2-\mathrm{C} 13$ & $-173.7(3)$ & $\mathrm{O} 3-\mathrm{C} 14-\mathrm{C} 15-\mathrm{C} 26$ & $-174.0(3)$ \\
\hline $\mathrm{O} 2-\mathrm{C} 1-\mathrm{C} 2-\mathrm{C} 3$ & $-171.3(3)$ & $\mathrm{O} 4-\mathrm{C} 14-\mathrm{C} 15-\mathrm{C} 16$ & $-172.2(3)$ \\
\hline $\mathrm{O} 2-\mathrm{C} 1-\mathrm{C} 2-\mathrm{C} 13$ & $6.6(5)$ & $\mathrm{O} 4-\mathrm{C} 14-\mathrm{C} 15-\mathrm{C} 26$ & $5.7(4)$ \\
\hline $\mathrm{C} 1-\mathrm{C} 2-\mathrm{C} 3-\mathrm{C} 4$ & $179.7(3)$ & $\mathrm{C} 14-\mathrm{C} 15-\mathrm{C} 16-\mathrm{C} 17$ & $-179.3(3)$ \\
\hline $\mathrm{C} 13-\mathrm{C} 2-\mathrm{C} 3-\mathrm{C} 4$ & $2.0(6)$ & $\mathrm{C} 26-\mathrm{C} 15-\mathrm{C} 16-\mathrm{C} 17$ & $3.1(5)$ \\
\hline $\mathrm{C} 2-\mathrm{C} 3-\mathrm{C} 4-\mathrm{C} 5$ & $-150.3(3)$ & $\mathrm{C} 15-\mathrm{C} 16-\mathrm{C} 17-\mathrm{C} 18$ & $-149.2(3)$ \\
\hline $\mathrm{C} 2-\mathrm{C} 3-\mathrm{C} 4-\mathrm{C} 9$ & $31.8(5)$ & $\mathrm{C} 15-\mathrm{C} 16-\mathrm{C} 17-\mathrm{C} 22$ & $33.4(5)$ \\
\hline $\mathrm{C} 3-\mathrm{C} 4-\mathrm{C} 5-\mathrm{C} 6$ & $179.8(3)$ & $\mathrm{C} 16-\mathrm{C} 17-\mathrm{C} 18-\mathrm{C} 19$ & $-179.4(3)$ \\
\hline $\mathrm{C} 9-\mathrm{C} 4-\mathrm{C} 5-\mathrm{C} 6$ & $-2.1(5)$ & $\mathrm{C} 22-\mathrm{C} 17-\mathrm{C} 18-\mathrm{C} 19$ & $-1.7(4)$ \\
\hline $\mathrm{C} 3-\mathrm{C} 4-\mathrm{C} 9-\mathrm{C} 8$ & $179.7(3)$ & $\mathrm{C} 16-\mathrm{C} 17-\mathrm{C} 22-\mathrm{C} 21$ & $178.4(3)$ \\
\hline $\mathrm{C} 5-\mathrm{C} 4-\mathrm{C} 9-\mathrm{C} 8$ & $1.7(5)$ & $\mathrm{C} 18-\mathrm{C} 17-\mathrm{C} 22-\mathrm{C} 21$ & $0.9(4)$ \\
\hline $\mathrm{C} 4-\mathrm{C} 5-\mathrm{C} 6-\mathrm{C} 7$ & $1.1(5)$ & $\mathrm{C} 17-\mathrm{C} 18-\mathrm{C} 19-\mathrm{C} 20$ & $2.1(5)$ \\
\hline $\mathrm{C} 5-\mathrm{C} 6-\mathrm{C} 7-\mathrm{C} 8$ & $0.4(5)$ & $\mathrm{C} 18-\mathrm{C} 19-\mathrm{C} 20-\mathrm{C} 21$ & $-1.6(5)$ \\
\hline $\mathrm{C} 5-\mathrm{C} 6-\mathrm{C} 7-\mathrm{C} 10$ & $179.4(3)$ & $\mathrm{C} 18-\mathrm{C} 19-\mathrm{C} 20-\mathrm{C} 23$ & $177.2(3)$ \\
\hline $\mathrm{C} 6-\mathrm{C} 7-\mathrm{C} 8-\mathrm{C} 9$ & $-0.8(5)$ & $\mathrm{C} 19-\mathrm{C} 20-\mathrm{C} 21-\mathrm{C} 22$ & $0.8(5)$ \\
\hline $\mathrm{C} 10-\mathrm{C} 7-\mathrm{C} 8-\mathrm{C} 9$ & $-179.8(3)$ & $\mathrm{C} 23-\mathrm{C} 20-\mathrm{C} 21-\mathrm{C} 22$ & $-177.9(3)$ \\
\hline $\mathrm{C} 6-\mathrm{C} 7-\mathrm{C} 10-\mathrm{C} 11$ & $-111.1(3)$ & $\mathrm{C} 19-\mathrm{C} 20-\mathrm{C} 23-\mathrm{C} 24$ & $50.7(4)$ \\
\hline $\mathrm{C} 6-\mathrm{C} 7-\mathrm{C} 10-\mathrm{C} 12$ & $123.4(3)$ & $\mathrm{C} 19-\mathrm{C} 20-\mathrm{C} 23-\mathrm{C} 25$ & $-72.6(4)$ \\
\hline $\mathrm{C} 8-\mathrm{C} 7-\mathrm{C} 10-\mathrm{C} 11$ & $68.0(4)$ & $\mathrm{C} 21-\mathrm{C} 20-\mathrm{C} 23-\mathrm{C} 24$ & $-130.6(3)$ \\
\hline
\end{tabular}




\section{supporting information}

$\begin{array}{llll}\mathrm{C} 8-\mathrm{C} 7-\mathrm{C} 10-\mathrm{C} 12 & -57.6(4) & \mathrm{C} 21-\mathrm{C} 20-\mathrm{C} 23-\mathrm{C} 25 & 106.1(4) \\ \mathrm{C} 7-\mathrm{C} 8-\mathrm{C} 9-\mathrm{C} 4 & -0.3(6) & \mathrm{C} 20-\mathrm{C} 21-\mathrm{C} 22-\mathrm{C} 17 & -0.6(5)\end{array}$

Symmetry codes: (i) $x, y, z+1$; (ii) $x, y, z-1$; (iii) $-x+1,-y+2,-z$; (iv) $-x+1,-y+1,-z$; (v) $-x,-y+2,-z$; (vi) $x-1, y, z$; (vii) $-x+2,-y+1,-z+1$; (viii) $x+1, y$, $z$.

Hydrogen-bond geometry $\left(A,{ }^{\circ}\right)$

\begin{tabular}{lllll}
\hline$D-\mathrm{H} \cdots A$ & $D-\mathrm{H}$ & $\mathrm{H} \cdots A$ & $D \cdots A$ & $D-\mathrm{H} \cdots A$ \\
\hline $\mathrm{O} 1-\mathrm{H} 1 \cdots \mathrm{O} 4^{\mathrm{i}}$ & $0.94(4)$ & $1.71(4)$ & $2.644(4)$ & $175(3)$ \\
$\mathrm{O} 3-\mathrm{H} 3 A \cdots \mathrm{O} 2^{\mathrm{ii}}$ & $0.93(4)$ & $1.71(4)$ & $2.631(3)$ & $169(3)$ \\
$\mathrm{C} 3-\mathrm{H} 3 \cdots \mathrm{O} 1$ & $0.96(3)$ & $2.35(2)$ & $2.707(4)$ & $101.2(16)$ \\
$\mathrm{C} 13-\mathrm{H} 13 A \cdots \mathrm{O} 2$ & 0.9600 & 2.2800 & $2.759(4)$ & 110.00 \\
$\mathrm{C} 16-\mathrm{H} 16 \cdots \mathrm{O} 3$ & $0.91(3)$ & $2.31(2)$ & $2.698(4)$ & $105.1(18)$ \\
$\mathrm{C} 26-\mathrm{H} 26 A \cdots \mathrm{O} 4$ & 0.9600 & 2.3000 & $2.770(4)$ & 110.00
\end{tabular}

Symmetry codes: (i) $x, y, z+1$; (ii) $x, y, z-1$. 